\title{
Biomedicine Hub
}

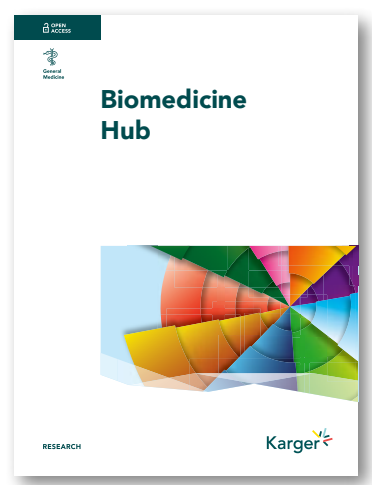

OP OPEN

General

Medicine

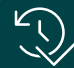

Time to final decision 13 weeks

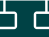

PMC

?

DOAJ

000

Registered Reports, Graphic abstracts

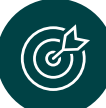

Visual abstract bmh@karger.com karger.com/bmh

Twitter
The interdisciplinary "hub" for researchers, clinicians, and public health professionals

Biomedicine Hub is an interdisciplinary Open Access journal: a "hub" for researchers, clinicians, and public health professionals across biomedical disciplines to share research findings, ideas, theories, experimental results, and clinical experience.

Biomedicine Hub facilitates the exchange of knowledge between disciplines, with a focus on translational science and emerging interdisciplinary topics. It encourages international collaborations and supports early-career researchers. By accepting Registered Reports in addition to classic article types, early feedback on research work is also guaranteed.

Biomedicine Hub publishes all relevant articles of sound ethical and scientific quality regardless of their novelty or expected impact.

Contributions from a wide range of biomedical fields are welcome. 